\title{
FAST RECURSION FORMULA FOR WEIGHT MULTIPLICITIES ${ }^{1}$
}

BY R. V. MOODY AND J. PATERA

The purpose of this note is to describe and prove a fast recursion formula for computing multiplicities of weights of finite dimensional representations of simple Lie algebras over $\mathbf{C}$.

Until now information about weight multiplicities for all but some special cases $[1,2]$ has had to be found from the recursion formulas of Freudenthal [3] or Racah [4]. Typically these formulas become too laborious to use for hand computations for ranks $\gtrsim 5$ and dimensions $\gtrsim 100$ and for ranks $\simeq 10$ and dimensions $\simeq 10^{4}$ on a large computer $[5,6]$. With the proposed method the multiplicities can routinely be calculated, even by hand, for dimensions far exceeding these. As an example we present a summary of calculations [7] of all multiplicities in the first sixteen irreducible representations of $E_{\mathbf{8}}$.

Let $(S$ be a semisimple Lie algebra over $\mathbf{C}$ with root system $\Delta$ and Weyl group $W$ relative to a Cartan subalgebra $\zeta$. Let $\Delta^{+}$be the positive roots with respect to some ordering and $\Pi=\left\{\alpha_{1}, \ldots, \alpha_{l}\right\}$ the set of simple roots. Let $Q$ and $P$ be the root and weight lattices respectively spanning the real vector space $V \subset$ G $^{*}$. If $X \subset P$ we denote by $X^{++}$the set of dominant elements of $X$ relative to $\Pi$.

Let $M$ be an irreducible (S)-module with highest weight $\Lambda$ and weight system $\Omega$. An important feature of the approach is the direct determination of $\Omega^{++}$without computing outside the dominant chamber. Since every $W$-orbit is represented by one weight $\lambda \in \Omega^{++}$of the same multiplicity, it suffices to compute such $\lambda$ 's.

The recursion formula for computing the multiplicities is a modification (Proposition 4) of the Freudenthal formula in which the Weyl group has been exploited to collapse it as much as possible. After describing the procedure, we present the $E_{\mathbf{8}}$ example. Finally the necessary proofs are given.

Received by the editors August 24, 1981.

1980 Mathematics Subject Classification. Primary 17B10; Secondary 22E46, 17 B20.

${ }^{1}$ Work supported in part by the National Science and Engineering Research Council of Canada and by the Ministère de l'Education du Québec.

(c) 1982 American Mathematical Society 0273-0979/82/0000-0254/\$02.50 
Computation of dominant weights and their multiplicities. Examples.

Determination of $\Omega^{++}$. We define inductively a set of disjoint subsets ('layers') of $P^{++}, L_{k}, k=0,1,2, \ldots$, by (1) $L_{0}=\{\Lambda\}, \quad L_{k}=\left\{\gamma \in P^{++}-L_{k-1} \mid \gamma=\lambda-\beta, \lambda \in L_{k-1}, \beta \in \Delta^{+}\right\}$. Then (Proposition 1) $\bigcup_{k=0}^{\infty} L_{k}=\Omega^{++}$. Thus $\Omega^{++}$can be found directly by computing the layers beginning with $L_{0}$. After $\Omega^{++}$is computed in this way it is reordered according to level. If $\rho^{2} \in V$ is defined by $\left(\rho^{2}, \alpha_{i}\right)=1$ for all $i$, then the new (partial) ordering of $\Omega^{++}$is given by the integers $\left(\lambda, \rho^{\vee}\right), \lambda \in \Omega^{++}$.

Computation of the multiplicity $m_{\lambda}$ of $\lambda \in \Omega^{++}$. An $m_{\lambda}$ of level $k$ is given in terms of the multiplicities $m_{\lambda^{\prime}}$ of weights $\lambda^{\prime}$ of levels above the $k$ th one.

Let $\operatorname{Stab}_{W}(\lambda)$ be the stabilizer of $\lambda$ in $W$. Then $\operatorname{Stab}_{W}(\lambda)=W_{T}:=\left\langle r_{i} \mid i \in T\right\rangle$, where $T=\left\{i \mid\left(\lambda, \alpha_{i}\right)=0\right\}[8]$. Let $\hat{W}_{T}=\left\langle W_{T},-1_{V}\right\rangle$, where $1_{V}$ is the identity transformation on $V$. $\hat{W}_{T}$ decomposes $\Delta$ into orbits $o_{1}, \ldots, o_{n}$. Each orbit $o_{i}$ contains a unique $\xi_{i}=\Sigma n_{i j} \omega_{j}, \xi_{i} \in \Delta^{+}$and $n_{i j} \geqslant 0$ for all $j \in T$ (Proposition 3). The modified Freudenthal formula is

$$
\sum_{i=1}^{n}\left|o_{i}\right| \sum_{p=1}^{\infty}\left(\lambda+p \xi_{i}, \xi_{i}\right) m_{\lambda+p \xi_{i}}=\left(c_{\Lambda}-c_{\lambda}\right) m_{\lambda},
$$

where $\left|o_{i}\right|$ is the number of elements of $o_{i}$ and, for all $\mu \in P$,

$$
c_{\mu}:=(\mu+\rho, \mu+\rho)-(\rho, \rho), \quad \rho:=\frac{1}{2} \sum_{\alpha \in \Delta^{+}} \alpha .
$$

The sum on $p$ is in reality finite and by standard properties of weight strings $\lambda+p \xi_{i} \notin \Omega \Rightarrow \lambda+q \xi_{i} \notin \Omega$ for $q>p$.

It is advantageous to work in the $\omega$-basis of the fundamental weights when computing $m_{\lambda}$. Thus writing $\lambda=\Sigma n_{i} \omega_{i}, T=\left\{i \mid n_{i}=0\right\}$. When the positive roots are expressed in this basis one easily determines $\xi_{i}$ 's. With $S_{i}:=$ $\left\{j \in T \mid\left(\xi_{i}, \alpha_{j}\right)=0\right\}$ the orbit sizes $\left|o_{i}\right|$ are given by subgroup indices $\left[W_{T}: W_{S_{i}}\right]$ or $2\left[W_{T}: W_{S_{i}}\right]$ (Proposition 3).

If in the relation (2) some weight $\mu=\lambda+p \xi_{i}=\Sigma n_{j} \omega_{j}$ is not in $P^{++}$, then some $n_{j}<0$ and $r_{j} \mu=\mu-n_{j} \alpha_{j}$ is on a higher level. A finite number $\left(\leqslant\left|\Delta^{+}\right|\right)$of reflections $r_{i}$ transforms $\mu$ into $\nu \in P^{++}$and $m_{\nu}$ is already computed.

If an extensive computation of weight multiplicities is to be undertaken, it is important to notice that for a given $(\$)$ there are only finitely many subsets $T$ of $\{1,2, \ldots, l\}$ and corresponding $\xi_{i}$ and $\left|o_{i}\right|$. It is natural to compute this information once and for all. We are preparing such a table.

Consider an example of the $E_{8}$ representation of dimension 4096000 . There are only nine weights in $\Omega^{++}$. In the basis of fundamental weights these are (after reordering according to levels) $\lambda_{0}, \ldots, \lambda_{8}$ (see Table). Here the layers are $L_{0}=\left\{\lambda_{8}\right\}, L_{1}=\left\{\lambda_{7}, \lambda_{6}, \lambda_{3}\right\}, L_{2}=\left\{\lambda_{5}, \lambda_{4}, \lambda_{2}, \lambda_{1}\right\}, L_{3}=\left\{\lambda_{0}\right\}$. Given $\Delta^{+}$in the $\omega$-basis, even by hand, the computation of (4) can be done in a matter of minutes. 


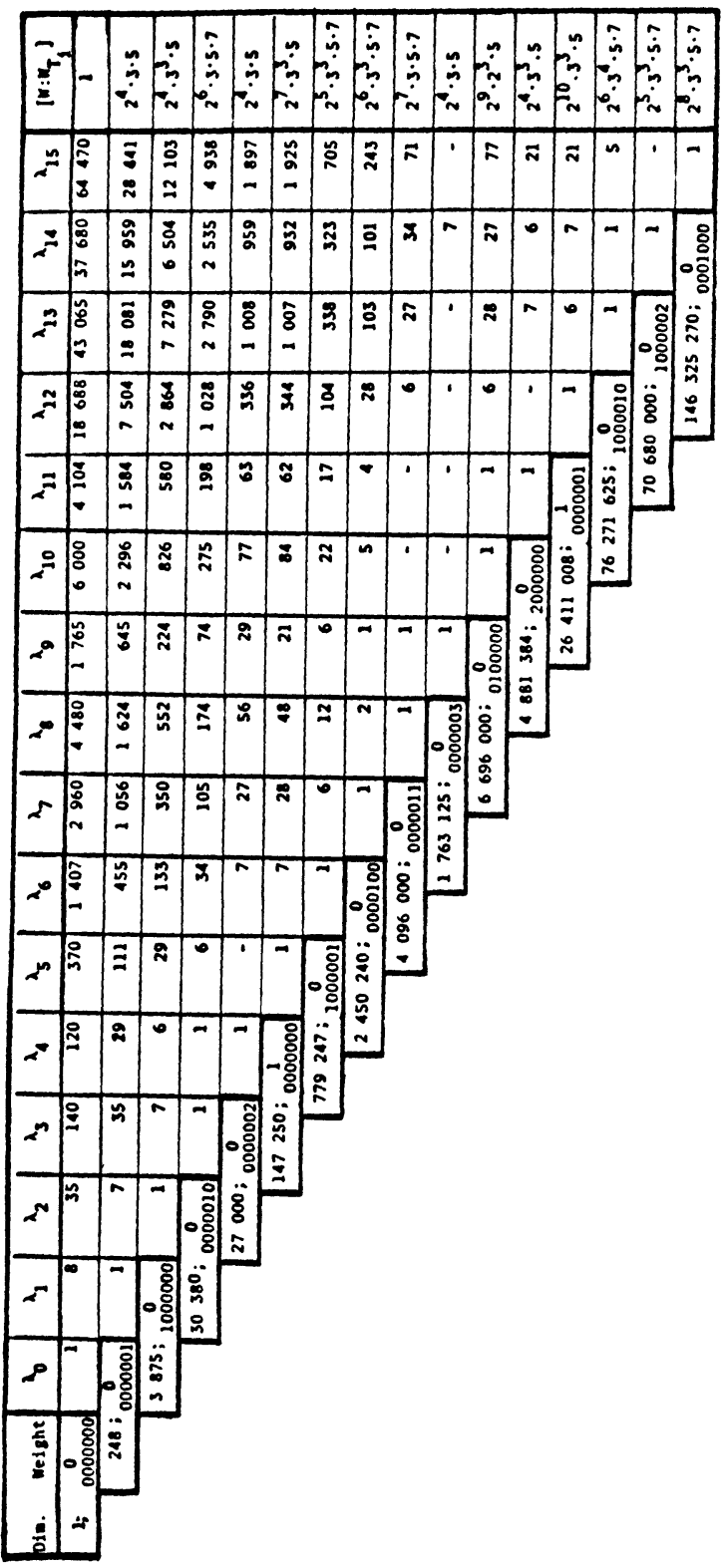

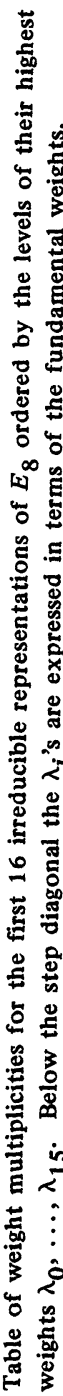

The determination of the multiplicity $m_{\lambda_{2}}$ is representative. Suppose we already know $m_{\lambda_{8}}=1, m_{\lambda_{7}}=2, m_{\lambda_{6}}=12, m_{\lambda_{5}}=48, m_{\lambda_{4}}=56, m_{\lambda_{3}}=174$. First we find the quantities $\xi_{i}$ and $\left|o_{i}\right|$ for $\lambda_{2}$. They are $\xi_{1}=0000001,\left|o_{1}\right|=28$, $\xi_{2}=-1000000,\left|o_{2}\right|=128, \xi_{3}={ }_{-1000010}^{0},\left|o_{3}\right|=84$. Next we find the weights $\lambda_{2}+p \xi_{i}$ which either are in $\Omega^{++}$or are transformed there by a sequence 
of reflections $r_{j}, j=1,2, \ldots, 8$. These weights are $\lambda_{2}+\xi_{1}=\lambda_{6}, \lambda_{2}+\xi_{2}=$ $\lambda_{5}, \lambda_{2}+\xi_{3}=\lambda_{3}$, and $\lambda_{2}+2 \xi_{3}=-1000020$ which after 16 reflections $r_{j}$ is transformed into $\lambda_{7}$. Hence (2) reads

$$
\begin{aligned}
\left|o_{1}\right|\left(\lambda_{6}, \xi_{1}\right) m_{\lambda_{6}} & +\left|o_{2}\right|\left(\lambda_{5}, \xi_{2}\right) m_{\lambda_{5}}+\left|o_{3}\right|\left(\lambda_{3}, \xi_{3}\right) m_{\lambda_{3}} \\
& +\left|o_{3}\right|\left(\lambda_{2}+2 \xi_{3}, \xi_{3}\right) m_{\lambda_{7}}=\left(c_{\lambda_{8}}-c_{\lambda_{2}}\right) m_{\lambda_{2}}
\end{aligned}
$$

Substituting the corresponding values into (4), one has $28 \cdot 4 \cdot 12+128 \cdot 3$. $48+84 \cdot 2 \cdot 174+84 \cdot 4 \cdot 2=(186-96) m_{\lambda_{2}}$ which gives $m_{\lambda_{2}}=552$.

The Table summarizes our results for the 16 irreducible representations of $E_{8}$. A useful check of the results is the equality of dimensions

$$
\operatorname{dim}(M)=\sum_{\lambda_{i} \in \Omega^{++}}\left[W: W_{T_{i}}\right] m_{\lambda_{i}},
$$

where the dimensions $\operatorname{dim}(M)$, the number $\left[W: W_{T_{i}}\right]$ of weights on each $W$-orbit, and the multiplicities $m_{\lambda_{i}}$ are given in the Table.

\section{Theory.}

Proposition 1. Let $M$ be an irreducible (S)-module with highest weight $\Lambda$. Then for $\lambda \in P^{++}$with $\lambda \neq \Lambda, \lambda \in \Omega^{++}$if and only if $\left(\lambda+\Delta^{+}\right) \cap \Omega^{++}$ $\neq \varnothing$.

Proof. Suppose that $\lambda \in P^{++}, \alpha \in \Delta^{+}$and $\mu:=\lambda+\alpha \in \Omega^{++}$. For all $\beta \in \Delta^{+},(\lambda, \alpha) \geqslant 0$. Then $(\mu, \alpha)=(\lambda+\alpha, \alpha)>0$. Since the weight string through $\mu$ is $\mu+q \alpha, \ldots, \mu, \ldots, \mu-p \alpha$ where $p-q=2(\mu, \alpha) /(\alpha, \alpha),[3]$, it follows that $p>0$ and hence $\lambda \in \Omega \cap P^{++}=\Omega^{++}$.

Conversely, suppose that $\lambda \in \Omega^{++}, \lambda \neq \Lambda$. We show that there is an $\alpha \in \Delta^{+}$with $\lambda+\alpha \in \Omega^{++}$. There is a $\beta \in \Delta^{+}$with $\lambda+\beta \in \Omega$. If $\lambda+\beta$ is dominant we are done. If not $\left(\lambda+\beta, \alpha_{j}\right)<0$ for some $j$ so by the argument above the $a_{j}$-weight string through $\lambda+\beta$ contains $\lambda+\beta+\alpha_{j}$. Also $\left(\lambda, \alpha_{j}\right) \geqslant 0$ since $\lambda \in P^{++}$so $\left(\beta, \alpha_{j}\right)<0$. Then $\beta+\alpha_{j}$ is a root, $\beta+\alpha_{j} \in \Delta^{+}$, and $\lambda+\beta+$ $\alpha_{j} \in \Omega$. We can replace $\beta$ by $\beta+\alpha_{j}$ in the above and repeat. The process cannot continue indefinitely, so the required $\alpha$ exists.

An interesting consequence of Proposition 1 is

Proposition 2 (Notation of Proposition 1). Let $k$ be the largest integer such that $L_{k} \neq \varnothing$. Then $L_{k}$ is a singleton $\{\omega\}$. Furthermore, $\omega$ depends only on $\Lambda \bmod 0$. In particular $0 \in \Omega$ if and only if $\Lambda \in Q$.

For $T \subset\{1,2, \ldots, l\}$, let $V_{T}:=\Sigma_{i \in T} \mathbf{R} \alpha_{i}$, and let $\Delta_{T}$ be the root system based on sub-Coxeter-Dynkin diagram corresponding to the vertices labelled by $T$. Then $V_{T} \cap \Delta=\Delta_{T}$. 
Proposition 3. Let $T \subset\{1, \ldots, l\}$ be any subset. Then each orbit o of $\hat{W}_{T}$ in $\Delta$ contains a unique element $\xi \in \Delta^{+}$of the form $\Sigma n_{i} \omega_{i}$ where $n_{i} \geqslant 0$ for all $i \in T$. Furthermore, if $S=\left\{i \in T \mid n_{i}=0\right\}$ then either $|o|=\left[W_{T}: W_{S}\right]$ if $\xi \in \Delta_{T}$, or $|o|=2\left[W_{T}: W_{s}\right]$ if $\xi \notin \Delta_{T}$.

Proof (Existence). Let $o$ be an orbit of $\hat{w}_{T}$ in $\Delta$. Choose $\xi=\Sigma n_{i} \omega_{i}$ $=\Sigma c_{i} \alpha_{i} \in o$ with $h t \xi:=\Sigma c_{i}$ maximal. Since $-1_{V} \in \hat{W}_{T}, \xi \in \Delta^{+}$. Since $h t\left(r_{i} \xi\right)$ $\leqslant h t(\xi)$ for all $i \in T$, we have $\left(\xi, \alpha_{i}\right) \geqslant 0$ hence $n_{i} \geqslant 0$, if $i \in T$.

(UNIQUENESS). Let $\pi: V \rightarrow V_{T}$ be the orthogonal projection onto $V_{T}=$ $\Sigma_{j \in T} \mathbf{R} \alpha_{j}$. Then $i \in T, v \in V,\left(\pi(v), \alpha_{i}\right)=\left(v, \alpha_{i}\right)$ from which it follows that $\pi$ is $W_{T}$-equivariant. Furthermore, $\pi$ is injective on any $W_{T}$-orbit in $V$ since $V_{T} \cap$ ker $\pi=0$. Thus there is a unique element on each $W_{T}$-orbit in $V$ whose projection is in the dominant chamber of $V_{T}$ under $W_{T}$. If $\mu=\Sigma u_{j} \omega_{j}$ is such an element then $\left(\mu, \alpha_{i}\right)=\left(\pi(\mu), \alpha_{i}\right) \geqslant 0$ for all $i \in T$, that is $u_{i} \geqslant 0$ for all $i \in T$.

Now let $\xi \in \Delta^{+}$. If $\hat{W}_{T} \xi=W_{T} \xi$ then $-\xi \in W_{T} \xi$ so $W_{T} \xi \cap \Delta^{-} \neq \varnothing$, from which $\xi \in \Delta_{T}^{+}$. Conversely, if $\xi \in \Delta_{T}^{+}$then $-\xi \in W_{T} \xi$ so $\hat{W}_{T} \xi=W_{T} \xi$.

Finally suppose that distinct $\xi$, $\xi^{\prime}$ satisfy the hypothesis of the Proposition and define the same $\hat{W}_{T}$-orbit. Then by the above $W_{T} \xi \neq W_{T} \xi^{\prime}$ so in fact $\hat{W}_{T} \xi$ $\neq W_{T} \xi, \xi \notin \Delta_{T}, W_{T} \xi \subset \Delta^{+}$and $\xi^{\prime} \in-W_{T} \xi \subset \Delta^{-}$, a contradiction. This proves the uniqueness. The statement on the orbit size is immediate.

Proposition 4 (Modified Freudenthal Formula). Let $M$ be the irreducible (乡-module of highest weight $\Lambda$ and let $\lambda \in \Omega(\Lambda)$. Let $W_{T}=\operatorname{Stab}_{W}(\lambda)$ and let $o_{1}, \ldots, o_{n}$ be the orbits of $\hat{W}_{T}$ in $\Delta$ Let $\xi_{i} \in o_{i}$ be as in Proposition 3. Then equation (2) holds.

Proof. We may suppose the indexing of the orbits is taken so that $o_{1} \cup \cdots \cup o_{m}=\Delta_{T}, 0 \leqslant m \leqslant n$. For $i \leqslant m, o_{i}=W_{T} \xi_{i}$ whereas for $i>m$, $o_{i}=W_{T} \xi_{i} \cup-W_{T} \xi_{i}$ (disjoint). Begin with the form of Freudenthal's formula [3, Equation 48.2]:

$$
c_{\Lambda} m_{\lambda}=\sum_{\alpha \in \Delta} \sum_{p=0}^{\infty}(\lambda+p \alpha, \alpha) m_{\lambda+p \alpha}+(\lambda, \lambda) m_{\lambda} .
$$

For $w \in W_{T},(\lambda+p w \alpha, w \alpha)=(w(\lambda+p \alpha), w \alpha)=(\lambda+p \alpha, \alpha)$ and $m_{\lambda+p w \alpha}=$ $m_{\lambda+p \alpha}$, so the double sum of the right-hand side of (6) may be rewritten as

$$
\begin{aligned}
& \sum_{i=1}^{m}\left|W_{T} \xi_{i}\right| \sum_{p=0}^{\infty}\left(\lambda+p \xi_{i}, \xi_{j}\right) m_{\lambda+p \xi_{i}} \\
& \quad+\sum_{i=m+1}^{n}\left|W_{T} \xi_{i}\right| \sum_{p=0}^{\infty}\left\{\left(\lambda+p \xi_{i}, \xi_{i}\right) m_{\lambda+p \xi_{i}}+\left(\lambda-p \xi_{i},-\xi_{i}\right) m_{\lambda-p \xi_{i}}\right\}
\end{aligned}
$$

Now for any $\alpha \in \Delta$ there is the relation $[3, \S 48]$

$$
\sum_{p=-\infty}^{\infty}(\lambda+p \alpha, \alpha) m_{\lambda+p \alpha}=0
$$


by which (7) becomes

$$
\begin{aligned}
& \sum_{i=1}^{m}\left|W_{T} \xi_{i}\right| \sum_{p=0}^{\infty}\left(\lambda+p \xi_{i}, \xi_{i}\right) m_{\lambda+p \xi_{i}} \\
& \quad+\sum_{i=m+1}^{n}\left|W_{T} \xi_{i}\right|\left\{2 \sum_{p=1}^{\infty}\left(\lambda+p \xi_{i}, \xi_{i}\right) m_{\lambda+p \xi_{i}}+\left(\lambda, \xi_{i}\right) m_{\lambda}\right\} .
\end{aligned}
$$

Collect the coefficients of $m_{\lambda}$ appearing in (9). In the first sum occur those $\xi_{i}$ which are in $\Delta_{T}$. Since $\left(\lambda, \alpha_{j}\right)=0$ for $j \in T,\left(\lambda, \xi_{i}\right)=0$ for $j=1, \ldots, m$. In the second sum we have $\Sigma_{i=m+1}^{n}\left|W_{T} \xi_{i}\right|\left(\lambda, \xi_{i}\right)=\Sigma_{\alpha \in \Delta^{+}-\Delta_{T}^{+}}(\lambda, \alpha)=$ $\Sigma_{\alpha \in \Delta^{+}}(\lambda, \alpha)=2(\lambda, \rho)$. Taking account of the $(\lambda, \lambda) m_{\lambda}$ appearing in (6) and $2\left|W_{T} \xi_{i}\right|=\left|o_{i}\right|$ for $i>m$, we arrive at

$$
c_{\Lambda} m_{\lambda}=\sum_{i=1}^{n}\left|o_{i}\right| \sum_{p=1}^{\infty}\left(\lambda+p \xi_{i}, \xi_{i}\right) m_{\lambda+p \xi_{i}}+(2(\lambda, \rho)+(\lambda, \lambda)) m_{\lambda}
$$

which proves the proposition.

The authors gratefully acknowledge the hospitality of the Aspen Center for Physics where this paper was written.

\section{REFERENCES}

1. H. Freudenthal, Zur Berechnung der Charaktere der halbfachen Lieschen Gruppen. II, Koninkl. Nederl. Akademie, Proceedings Ser. A 57, No. 5 (1954), 487-491.

2. R. C. King and H. H. Al-Qubanchi, The Weyl group and weight multiplicities of the exceptional Lie groups, J. Phys. A 14 (1981), 51-75.

3. H. Freudenthal and H. deVries, Linear Lie groups, Academic Press, New York, 1969.

4. B. Kolman and J. A. Belinfante, Survey of Lie groups and Lie algebras with applications and computational methods, SIAM, Philadelphia, 1972.

5. V. K. Agrawala and J. G. Belinfante, Weight diagrams for Lie group representations: A computer implementation of Freudenthal's algorithm in ALGOL and FORTRAN, BIT 9 (1969), 301-304.

6. W. McKay, J. Patera and D. Sankoff, The computation of branching rules for representations of semisimple Lie algebras, Computers in Non-Associative Rings and Algebras (R. Beck and B. Kolman, eds.), Academic Press, New York, 1977.

7. M. Bremner, R. Funk and R. Moody, Implementation of the fast recursion formula in PASCAL.

8. N. Bourbaki, Groupes et algèbres de Lie, Chapitres 4-6, Hermann, Paris, 1968.

DEPARTMENT OF MATHEMATICS, UNIVERSITY OF SASKATCHEWAN, SASKATOON, SASKATCHEWAN, CANADA S7N OWO

CENTRE DE RECHERCHE DE MATHÉMATIQUES APPLIQUÉES, UNIVERSITÉ DU MONTRÉAL, QUÉBEC, CANADA H3C 3J 7 